\title{
The Impact of Lower Extremity Venous Ulcers due to Chronic Venous Insufficiency on Quality of Life
}

\author{
Sotirios A. Koupidis ${ }^{1}$ Kosmas I. Paraskevas $^{2}$ Vassilios Stathopoulos ${ }^{3}$ and Dimitri P. Mikhailidis ${ }^{*} 4$ \\ ${ }^{1}$ Athens University Medical School, Athens, Greece; ${ }^{2}$ Department of Vascular Surgery, Red Cross Hospital, Athens, \\ Greece; ${ }^{3} 2^{\text {nd }}$ Department of Surgery, "Agia Olga" Hospital, Athens, Greece; ${ }^{4}$ Department of Clinical Biochemistry \\ (Vascular Disease Prevention Clinics), Royal Free Hospital Campus, University College Medical School, University \\ College London (UCL), London, UK
}

\begin{abstract}
Lower extremity venous ulcers comprise a complex medical and social issue. The conservative and/or surgical management of venous ulcers is often inadequate. In addition, the psychosocial aspect of the disease is often overlooked and most often undertreated. Common symptoms such as pain, low self-esteem and patient isolation are usually not recognized and therefore not adequately managed.

This mini-review summarizes the current data on the management of lower extremity venous ulcers and their impact on the quality of life of these patients.
\end{abstract}

Key Words: Venous leg ulcers, quality of life, lower extremity venous ulcers.

\section{INTRODUCTION}

Chronic venous insufficiency of the lower extremities affects approximately 2.5 million people in the USA and has an important effect on daily activities and quality of life [13]. Besides pain, swelling, edema, varicosity of the superficial veins and skin changes, chronic venous insufficiency is associated with venous leg ulcers, a condition that requires a multidisciplinary and aggressive therapeutic approach [2$10]$. Nearly 500,000 individuals have painful venous ulcers [3].

Pain is a feature of lower extremity venous ulcers which is often overlooked and commonly undertreated [2, 3, 8, 9]. Furthermore, psychosocial issues such as anxiety and depression, a low self-esteem and social deprivation are quite common in these patients; these issues are often not adequately addressed $[2,3,8,9]$.

This mini-review considers the impact of lower extremity venous ulcers on quality of life.

\section{QUALITY OF LIFE IN PATIENTS WITH VASCULAR LEG ULCERS}

A multi-centre, cross-sectional study assessed pain and quality of life in patients with chronic vascular leg ulcers by use of a questionnaire that incorporated the Short Form (SF)12 health survey and a visual analogue scale (VAS) for the assessment of pain during the day, night and at dressing change [11]. Women experienced more pain and had a worse quality of life than men. Venous ulcers had high mean VAS values during the day and night (44.4 and 44.9, respectively). However, a higher mean value was recorded at dressing change, which was similar to the mean VAS value for vascular

*Address correspondence to this author at the Department of Clinical Biochemistry (Vascular Disease Prevention Clinics), Royal Free Hospital Campus, Pond street, London NW3 2QG, UK; E-mail: MIKHAILIDIS@aol.com ulcers ( 57.5 vs 56.3, respectively). There was a direct correlation between pain and quality of life, which was worse for ulcers with a longer duration and larger area. Ulcers of $<3$ months' duration had mean VAS values of 38.7, 36.9, 46.8 compared with 53.8, 53.9, 62.9 for ulcers of >12 months' duration. Mean SF-12 values for the physical component of the questionnaire also showed that patients whose ulcers had a longer duration had a poorer quality of life than patients whose ulcers were more recent (35.9 vs 40.9, respectively). The conclusion reached was that vascular leg ulcers result in a poor quality of life, with a direct correlation with both ulcer duration and ulcer area [11].

Besides pain, lower extremity venous ulcers also have an effect on patient self-esteem and social life. In a study from the UK, a questionnaire was distributed to 196 people with chronic lower extremity ulcers [12]. This questionnaire aimed to determine the impact of odour and excessive exudate leading to leakage on patient quality of life. These symptoms had an adverse effect on the psychological state, leading to feelings of disgust, self-loathing and low selfesteem. The net result was social isolation and depression [12].

In another study, 38 patients with venous leg ulcers completed a health-related quality of life questionnaire (modified Skindex) and were then interviewed by a psychologist using a semi-structured guide [13]. Data from the questionnaire were used to evaluate the impact of venous leg ulcers on patients' lives. Interview transcripts were analysed using qualitative methods to identify additional venous leg ulcerspecific health-related quality of life items. The Skindex scores showed that older patients had worse health-related quality of life results $(p<0.05)$, as did those with pain and non-healing ulcers. Ulcer duration and size did not correlate with quality of life. Based on the results of the interviews, pain $(80.5 \%)$, itching $(69.4 \%)$, altered appearance $(66.7 \%)$, loss of sleep (66.6\%), functional limitation $(58.3 \%)$ and dis- 
appointment with treatment $(50 \%)$ were identified as the psychological effects of venous leg ulcers [13].

A recent evaluation study of venous leg ulcer guidelines concluded that all lower extremity ulcer practice guidelines are evidence-based [14]. There is often a lack of patient perspective and use of a uniform method to weigh the evidence. In addition, limited attention is given to pain and lifestyle advice recommendations. Most of the guidelines fail to consider the issues of dissemination and implementation; furthermore, revisions of the guidelines are often not available. The authors recommend that leg ulcer guidelines should incorporate a multidisciplinary approach and they underline the importance of patient involvement. Finally, recommendations on pain, lifestyle advice, compliance and other quality-of-life issues should be incorporated in guidelines for leg ulcer treatment [14].

\section{CONSERVATIVE VS SURGICAL TREATMENT OPTIONS FOR VENOUS LEG ULCERS}

The use of compression stockings, as well as proper wound and skin care are the mainstay of conservative treatment of venous leg ulcers [15, 16]. Use of compression stockings with $30-40 \mathrm{mmHg}$ of tension results in significant improvement in pain, swelling, skin pigmentation, activity and patient satisfaction, provided a $70 \%-80 \%$ compliance to compression therapy is achieved [15]. With a structured regimen of compression therapy more than 9 out of 10 patients with venous ulcers can achieve complete healing in less than half a year (mean time: 5.3 months) [16].

In an 8-week randomised comparison of a 2-layer (Coban 2 Layer) and a 4-layer (Profore) compression bandage system for venous ulcers, the superiority of the former over the latter was prominent [17]. The primary endpoint was bandage slippage measured at each dressing change while secondary endpoints included patient preference, wound healing and health-related quality of life. The 2-layer system showed less bandage slippage compared with the 4-layer system (mean slippage: 2.48 vs $4.17 \mathrm{~cm}$, respectively; $p<0.001$ ). The health-related quality of life Physical Symptoms and Daily Living scores were also higher for the 2-layer system (pooled 2-sample t-test; $p<0.05$ ). Most patients preferred the 2-layer over the 4-layer system ( $72 \%$ vs $22 \%$, respectively), with the remaining $6 \%$ having no preference. Finally, there were no differences in the percent of wounds that healed $(p=0.30)$, in wound area reduction $(p=0.88)$ or in linear healing rate $(p=0.94)$. These results indicated that, while less bandage slippage did not appear to have an impact on wound healing, it may have influenced patient preference in favour of the 2-layer system and possibly their healthrelated quality of life [17].

Another study investigated the impact of venous leg ulcers and effective compression treatment on quality of life in 65 patients by use of the SF-36 quality-of-life questionnaire [18]. Data analysis included an investigation of the study population as whole, differences between patients whose ulcers did and did not heal and between gender and age. This study showed that there was a significant improvement in the SF-36 domains of bodily pain, health transition, mental health and social functioning for all 65 patients. Patients whose ulcers healed also showed a considerable improvement in the vitality domain. Even patients whose ulcers did not heal had improved scores for bodily pain and health transition. These results demonstrated that good wound management and effective compression therapy can improve quality of life in patients with venous leg ulceration, whether or not the patient's leg ulcer healed following treatment [18].

A Cochrane review and meta-analysis was recently published including randomised controlled trials either comparing intermittent pneumatic compression (IPC) with control (Sham IPC or no IPC) or comparing IPC treatment regimens in venous ulcer management [19]. Seven randomised controlled trials ( $n=367$ patients) were identified. Only 1 trial reported both allocation concealment and blinded outcome assessment. In 1 trial ( $\mathrm{n}=80$ patients) more ulcers healed with IPC than with dressings $(62 \%$ vs $28 \%$, respectively; $p=$ 0.002). Additionally, 4 trials compared IPC with compression against compression alone. The first of these trials ( $n=45$ study participants) found increased ulcer healing with IPC plus compression than with compression alone (relative risk for healing: $11.4 ; 95 \%$ confidence interval, 1.6-82). The remaining 3 trials ( $n=122$ patients) found no evidence of a benefit for IPC plus compression compared with compression alone. One small trial $(n=16$ patients $)$ found no difference between IPC (without additional compression) and compression bandages alone. Another trial compared different ways of delivering IPC ( $n=104$ patients) and found that rapid IPC healed more ulcers compared with slow IPC (86\% vs $61 \%$, respectively; log-rank $p=0.003)$. The conclusion reached was that, compared with no compression, IPC may increase healing but whether it increases healing when added to treatment with bandages, or if it can be used instead of compression bandages is not clear [19]. Further trials are required to determine whether IPC increases the healing of venous leg ulcers when used in modern practice where compression therapy is widely used.

A systematic review showed that while lifestyle is mentioned in most leg ulcer guidelines, limited emphasis is paid on this subject [20]. This article considered the published evidence for the effect of nutrition, leg elevation and exercise on the healing of leg ulcers, as well as the effect of painrelated interventions. While some evidence was found to support a positive effect of leg exercises on the endurance and power of the calf muscle and on the haemodynamic status of the limb, as well as a positive effect of leg elevation during bed rest without compression, no hard evidence was found concerning the effect of enriched or altered nutrition on wound healing. Nevertheless, there was some evidence of nutritional deficits in this patient group. In addition, while the use of eutectic mixture of local anaesthetic cream is effective for reducing pain in wound debridement, pain relief in daily life is insufficiently treated. The conclusion reached was that, although there is no hard evidence, it can be expected that interventions such as nutritional monitoring, guided exercise and leg elevation will have a substantial impact on wound healing in patients with venous leg ulceration [20].

The pharmacological management of lower extremity venous ulcers due to chronic venous insufficiency is a complex procedure that needs to address several issues. Progressive chronic venous insufficiency may lead to compromised skin integrity; it is thus important to keep the area wellmoisturized to reduce skin breakdown and concomitant in- 
fections [21]. Use of steroids in cases of stasis dermatitis, as well as aggressive wound care and application of appropriate antibiotics in cases of venous ulcers and superimposed bacterial infections are additional measures [21].

In addition, several therapeutic approaches have been proposed. For example, local application of a hydrogen peroxide cream (e.g. Crystacide) in patients with chronic venous insufficiency, venous hypertension and skin ulcerations reduced the ulcerated areas and improved skin perfusion compared with non-use [22]. Crystacide application improved transcutaneous $\mathrm{PO}_{2}$ and decreased $\mathrm{PCO}_{2}$, free radical production and laser Doppler flowmetry compared with nonuse. Similar positive results were reported after oral administration of oxerutins (Venoruton) [23], the endothelium activator inhibitor and anti-edematous micronised purified flavonoid fraction (Daflon) [24, 25] or Pycnogenol [26]. The effects of Pycnogenol in particular appear to extend to other vascular disorders as well [27-29]. Nevertheless, a systematic review of several oral or topical phlebotonics used (e.g. rutosides, calcium dobesilate, disodium flavodate, hidrosmine, diosmine, centella asiatica, chromocarbe, French maritime pine bark extract, grape seed extract and aminaftone) in patients with chronic venous insufficiency concluded that there is not yet enough evidence to strongly support their efficacy in such patients [30].

The effect of several other agents on lower extremity venous ulcers has also been evaluated. For example, the use of horsechestnut seed extract therapy is an effective, as well as cost-effective, alternative treatment for the management of venous leg ulcers [31-33]. A similar effect has been advocated for the tumor necrosis factor (TNF)-alpha antibody infliximab [34].

Besides these pharmacological options, several surgical approaches have been described for the management of chronic venous leg ulcers. Surgical intervention with regards to the deep venous system in the form of venous bypass or valve replacement remains disappointing [3, 35]. External venous valvuloplasty is an alternative surgical approach yielding comparable results $[36,37]$. A novel approach includes the use of stenting for the treatment of chronic venous insufficiency, which forms the basis for the development of venous leg ulcers [38-40]. The results of both in vitro [38] and in vivo [40] studies are promising. Verification of these preliminary results in large-scale trials holds implications for this treatment.

The traditional means of managing the superficial venous system when diseased has been to remove it in the forms of disconnecting the great or short saphenous veins from the deep system at the saphenofemoral or saphenopopliteal junctions, respectively. It is well established that stripping the long saphenous vein to below the knee at the same time reduces recurrence rates, but there is growing evidence that this may also be the case for the short saphenous vein. The assumed benefit of these procedures with regards to treating chronic venous insufficiency has only recently been verified in formal trials [41, 42]; although superficial venous surgery plus compression therapy does not appear to improve ulcer healing rates when compared with compression therapy alone, it is associated with a significantly lower ulcer recurrence rate [41-43].
The surgical management of incompetent venous perforator communications between the deep and superficial venous systems in the leg can be successfully undertaken by a subfascial endoscopic method, by laser ablation or foam sclerosant exclusion [44-46]. However, there is not enough evidence to support that this improves the rate of ulcer healing or reduces the rate of ulcer recurrence compared with external compression [44-46].

Debridement of the ulcer wound and addition of a skin graft to stimulate healing was proposed as an effective treatment of leg ulcers resistant to compression therapy [47, 48]. Skin grafts are divided into autografts (from the patient's own uninjured skin), allograft (bioengineered skin grown from donor cells) and xenografts (preserved skin from other animals, e.g. pigs). Although preliminary results seem promising, there is as yet not enough evidence to determine which types of skin grafting should comprise the treatmentof-choice for leg ulcers resistant to compression therapy [47, 48].

A study was undertaken to assess the effectiveness of skin grafting and the impact on the quality of life of patients with chronic venous ulcers [49]. Two groups of patients were involved in the study; one was treated by performing skin graft surgery, while the other group was treated only by conservative means. Patients had to complete a questionnaire that was used to evaluate the impact of chronic venous leg ulcers on their quality of life. At the beginning of the study all patients in both groups suffered from leg pain. At the end of the study, the pain had decreased in patients undergoing skin graft surgery, while in the group only receiving conservative treatment, the pain practically remained the same. Furthermore, at the beginning of the study, the ulcers deprived many patients $(37-41 \%)$ from both groups of sleep for most of the night. At the end of the study, ulcers only deprived $2.5 \%$ of patients in the surgical group for most of the night, whereas in the conservative group this percentage was reduced to only $20 \%$. The conclusion reached was that skin grafting positively influences the quality of life, decreases the limitation of function and improves the dysphoric mood. The coverage of ulcers by autografts of skin thickness decreases leg pain within 6 months compared with conservative treatment alone $(p<0.05)$ [49].

An earlier study from the same group compared the functionality of surgical treatment with conservative measures for the management of venous leg ulcers [50]. A total of 44 patients with large trophic leg ulcers (mean size $=254 \mathrm{~cm}^{2}$ ) were managed for $\geq 6$ months; 10 patients were treated conservatively and 34 patients were treated by skin grafting. All of them were interviewed after 3-6 months. Ulcer pain had decreased for the patients treated surgically whereas it remained the same for those treated conservatively. Initially, all patients $(n=44)$ had sleep disorders because of the ulcers. In the group of surgically-treated patients, ulcers did not disturb sleep 3 months following surgery, whereas the problem persisted in the group treated conservatively. A considerable improvement in the patients' emotional status was also noted [50].

\section{CONCLUSIONS}

The appropriate management of venous leg ulcers is complex and not fully defined. Venous ulcers have a consid- 
erable impact on patients' well-being and negatively affect their quality of life. Besides the establishment of the treatment-of-choice for this disease, it is equally important to address the psychosocial issues that usually accompany the development of lower extremity venous ulcers. Future studies should include this important parameter when evaluating the efficacy of a proposed treatment strategy.

\section{REFERENCES}

[1] Chiesa R, Marone EM, Limoni C, Volonté M, Schaefer E, Petrini O. Effect of chronic venous insufficiency on activities of daily living and quality of life: correlation of demographic factors with duplex ultrasonography findings. Angiology 2007; 58: 440-9.

[2] White JV, Ryjewski C. Chronic venous insufficiency. Perspect Vasc Surg Endovasc Ther 2005; 17: 319-27.

[3] Eberhardt RT, Raffeto JD. Chronic venous insufficiency. Circulation 2005; 111: 2398-409.

[4] Kantor J, Margolis D. Management of leg ulcers. Semin Cutan Med Surg 2003; 22: 212-21.

[5] Bongiovanni CM, Hughes MD, Bomengen RW. Accelerated wound healing: multidisciplinary advances in the care of venous leg ulcers. Angiology 2006; 57: 139-44.

[6] O'Meara S, Al-Kurdi D, Ovington LG. Antibiotics and antiseptics for venous leg ulcers. Cochrane Database Syst Rev 2008; 1: CD003557.

[7] Al-Kurdi D, Bell-Syer SE, Flemming K. Therapeutic ultrasound for venous leg ulcers. Cochrane Database Syst Rev 2008; 1: CD001180.

[8] Barron GS, Jacob SE, Kirsner RS. Dermatologic complications of chronic venous disease: medical management and beyond. Ann Vasc Surg 2007; 21: 652-62.

[9] Herschthal J, Kirsner RS. Current management of venous ulcers: an evidence-based review. Surg Technol Int 2008; 17: 77-83.

[10] Heinen MM, Persoon A, van de Kerkhof P, Otero M, van Achterberg T. Ulcer-related problems and health care needs in patients with venous leg ulcerations: a descriptive, cross-sectional study. Int J Nurs Stud 2007; 44: 1296-303.

[11] Guarnera G, Tinelli G, Abeni D, Di Pietro C, Sampogna F, Tabolli S. Pain and quality of life in patients with vascular leg ulcers: an Italian multicentre study. J Wound Care 2007; 16: 347-51.

[12] Jones JE, Robinson J, Barr W, Carlisle C. Impact of exudates and odour from chronic venous leg ulceration. Nurs Stand 2008; 22: 534.

[13] Hareendran A, Bradbury A, Budd J, et al. Measuring the impact of venous leg ulcers on quality of life. J Wound Care 2005; 14: 53-7.

[14] Van Hecke A, Grypdonck M, Defloor T. Guidelines for the management of venous leg ulcers: a gap analysis. J Eval Clin Pract 2008; In process citation

[15] Motykie GD, Caprini JA, Arcelus JI, Reyna JJ, Overom E, Mokhtee D. Evaluation of therapeutic compression stockings in the treatment of chronic venous insufficiency. Dermatol Surg 1999; 25: 116-20.

[16] Mayberry JC, Moneta GL, Taylor LM Jr, Porter JM. Fifteen-year results of ambulatory compression therapy for chronic venous ulcers. Surgery 1991; 109: 575-81.

[17] Moffatt CJ, Edwards L, Collier M, et al. A randomised controlled 8 -week crossover clinical evaluation of the $3 \mathrm{M}$ coban 2 layer compression system versus profore to evaluate the product performance in patients with venous leg ulcers. Int Wound J 2008; 5: 267-79.

[18] Charles H. Does leg ulcer treatment improve patients' quality of life? J Wound Care 2004; 13: 209-13.

[19] Nelson EA, Mani R, Vowden K. Intermittent pneumatic compression for treating venous leg ulcers. Cochrane Database Syst Rev 2008; 12: CD001899.

[20] Heinen MM, van Achterberg T, op Reimer WS, van de Kerkhof PC, de Laat E. Venous leg ulcer patients: a review of the literature on lifestyle and pain-related interventions. J Clin Nurs 2004; 13: 355-66.

[21] Felty CL, Rooke TW. Compression therapy for chronic venous insufficiency. Semin Vasc Surg 2005; 18: 36-40.

[22] Belcaro G, Cesarone MR, Errichi BM, et al. Improvement of microcirculation and healing of venous hypertension and ulcers with Crystacide: evaluation with a microcirculatory model, including free radicals, laser doppler flux, and $\mathrm{PO} / \mathrm{PCO} 2$ measurements. Angiology 2007; 58: 323-8.

[23] Cesarone MR, Belcaro G, Pellegrini L, et al. Venoruton vs Daflon: evaluation of effects on quality of life in chronic venous insufficiency. Angiology 2006; 57: 131-8.

[24] Katsenis K. Micronized purified flavonoid fraction (MPFF): a review of its pharmacological effects, therapeutic efficacy and benefits in the management of chronic venous insufficiency. Curr Vasc Pharmacol 2005; 3: 1-9.

[25] Bergan JJ. Chronic venous insufficiency and the therapeutic effects of Daflon $500 \mathrm{mg}$. Angiology 2005; 56 (Suppl 1): S21-4.

[26] Cesarone MR, Belcaro G, Rohdewald P, et al. Rapid relief of signs/symptoms in chronic venous microangiopathy with pycnogenol: a prospective, controlled study. Angiology 2006; 57: 569-76.

[27] Cesarone MR, Belcaro G, Rohdewald P, et al. Improvement of diabetic microangiopathy with pycnogenol: A prospective, controlled study. Angiology 2006; 57: 431-6.

[28] Vinciguerra G, Belcaro G, Cesarone MR, et al. Cramps and muscular pain: prevention with pycnogenol in normal subjects, venous patients, athletes, claudicants and in diabetic microangiopathy. Angiology 2006; 57: 331-9.

[29] Cesarone MR, Belcaro G, Pellegrini L, et al. HR, 0-(betahydroxyethyl)-rutosides; (Venoruton): rapid relief of signs/symptoms in chronic venous insufficiency and microangiopathy: a prospective, controlled study. Angiology 2005; 56: 165-72.

[30] Martinez MJ, Bonfill X, Moreno RM, Vargas E, Capellà D. Phlebotonics for venous insufficiency. Cochrane Database Syst Rev 2005; 20:CD003229.

[31] Leach MJ, Pincombe J, Foster G. Using horsechestnut seed extract in the treatment of venous leg ulcers: a cost-benefit analysis. Ostomy Wound Manage 2006; 52: 68-70, 72-4, 76-8.

[32] Leach MJ, Pincombe J, Foster G. Clinical efficacy of horsechestnut seed extract in the treatment of venous ulceration. J Wound Care 2006; 15: 159-67.

[33] Suter A, Bommer S, Rechner J. Treatment of patients with venous insufficiency with fresh plant horse chestnut seed extract: a review of 5 clinical studies. Adv Ther 2006; 23: 179-90.

[34] Streit M, Beleznay Z, Braathen LR. Topical application of the tumour necrosis factor-alpha antibody infliximab improves healing of chronic wounds. Int Wound J 2006; 3: 171-9.

[35] Welch HJ. Surgical options for the treatment of venous ulcers. Vasc Endovascular Surg 2004; 38: 195-202.

[36] Rosales A, Slagsvold CE, Kroese AJ, Stranden E, Risum $\varnothing$, Jørgensen JJ. External venous valve plasty (EVVP) in patients with primary chronic venous insufficiency (PCVI). Eur J Vasc Endovasc Surg 2006; 32: 570-6.

[37] Wang SM, Hu ZJ, Li SQ, Huang XL, Ye CS. Effect of external valvuloplasty of the deep vein in the treatment of chronic venous insufficiency of the lower extremity. J Vasc Surg 2006; 44: 1296300.

[38] Geselschap JH, van Zuiden JM, Toonder IM, Wittens CH. In vitro evaluation of a new autologous valve-stent for deep venous incompetence. J Endovasc Ther 2006; 13: 762-9.

[39] Mussa FF, Peden EK, Zhou W, Lin PH, Lumsden AB, Bush RL. Iliac vein stenting for chronic venous insufficiency. Tex Heart Inst J 2007; 34: 60-6.

[40] Neglen P, Hollis KC, Raju S. Combined saphenous ablation and iliac stent placement for complex severe chronic venous disease. J Vasc Surg 2006; 44: 828-33.

[41] Gohel MS, Barwell JR, Wakely C, et al. The influence of superficial venous surgery and compression on incompetent calf perforators in chronic venous leg ulceration. Eur J Vasc Endovasc Surg 2005; 29: 78-82.

[42] Gohel MS, Barwell JR, Earnshaw JJ, et al. Randomized clinical trial of compression plus surgery versus compression alone in chronic venous ulceration (ESCHAR study)--haemodynamic and anatomical changes. Br J Surg 2005; 92: 291-7.

[43] Puggioni A, Kalra M, Gloviczki P. Superficial vein surgery and SEPS for chronic venous insufficiency. Semin Vasc Surg 2005; 18: 41-8.

[44] Baron HC, Wayne MG, Santiago C, et al. Treatment of severe chronic venous insufficiency using the subfascial endoscopic perforator vein procedure. Surg Endosc 2005; 19: 126-9.

[45] Puggioni A, Kalra M, Gloviczki P. Superficial vein surgery and SEPS for chronic venous insufficiency. Semin Vasc Surg 2005; 18: 41-8. 
[46] Bergan JJ, Pascarella L. Severe chronic venous insufficiency: primary treatment with sclerofoam. Semin Vasc Surg 2005; 18: 49-56.

[47] Jones JE, Nelson EA. Skin grafting for venous leg ulcers. Cochrane Database Syst Rev 2007; 2: CD001737.

[48] Marston W. Evaluation and treatment of leg ulcers associated with chronic venous insufficiency. Clin Plast Surg 2007; 34: 717-30.
[49] Jankunas V, Bagdonas R, Samsanavicius D, Rimdeika R. The influence of surgical treatment for chronic leg ulcers on the quality dynamics of the patient's life. Acta Chir Belg 2007; 107: 386-96.

[50] Jankūnas V, Rimdeika R, Jasenas M, Samsanavicius D. Changes in patient's quality of life comparing conservative and surgical treatment of venous leg ulcers. Medicina (Kaunas) 2004; 40: 731-9.

(C) Koupidis et al.; Licensee Bentham Open.

This is an open access article licensed under the terms of the Creative Commons Attribution Non-Commercial License (http://creativecommons.org/licenses/by$\mathrm{nc} / 3.0 /$ ) which permits unrestricted, non-commercial use, distribution and reproduction in any medium, provided the work is properly cited. 